\title{
Gastric and intestinal phenotypic marker expression in gastric carcinomas and recurrence pattern after surgery -immunohistochemical analysis of 213 lesions-
}

\author{
Y Tajima*,', K Yamazaki', N Nishino', K Morohara', T Yamazaki', T Kaetsu', S Suzuki', M Kawamura', \\ K Kumagai ${ }^{2}$ and $M$ Kusano'
}

'Department of Surgery, Division of General \& Gastroenterological Surgery, Showa University, School of Medicine, 1-5-8, Hatanodai, Shinagawa-ku, Tokyo 142-8666, Japan; ²Department of Surgery, Toyosu Hospital, Showa University, I-I 8, Toyosu 4 chome, Koto-ku, Tokyo I 35-8577, Japan

Both gastric and intestinal phenotypic markers are known to be expressed in gastric carcinomas, irrespective of their histologic type. In the present study, the relation between gastric and intestinal phenotypic marker expression in gastric carcinomas and the recurrence pattern after surgery was examined. The phenotypic marker expression of the tumour was determined by examining the expression of human gastric mucin (HGM), MUC6, MUC2 and CDI 0 in 213 advanced gastric carcinomas in 213 patients who had undergone a curative resection (97 died from recurrent gastric carcinoma and I 16 were alive without recurrence at the end of the follow-up period). Tumours were classified into gastric (G), gastric and intestinal mixed (GI), intestinal (I) or unclassified (UC) phenotypes according to the immunopositivity of HGM, MUC6, MUC2 and CD I 0 stainings. The incidence of HGM-positive tumours and MUC2-negative tumours was significantly higher in tumours with peritoneal recurrence than in tumours without recurrence (73.3\%, 44 out of 60 cases vs 54.3\%, 63 out of II6 ( $P=0.022)$; and 70.0\%, 42 out of 60 vs 38.8\%, 45 out of I I6 (P=0.0002), respectively). The incidence of G-phenotype tumours was also significantly higher in tumours with peritoneal recurrence than in tumours without recurrence $(58.3 \%, 35$ out of 60 cases vs 28.4\%, 33 out of $116(P=0.0002)$ ). The incidence of MUC2-negative tumours and CDI0-positive tumours was significantly higher in tumours with haematogenous recurrence than in tumours without recurrence (62.5\%, 20 out of 32 cases vs 38.8\%, 45 out of II6 $(P=0.028)$; and $43.8 \%$, I4 out of 32 vs $23.3 \%, 27$ out of II6 $(P=0.039)$; respectively). Our present findings show that the gastric and intestinal phenotypic marker expression of the tumour, determined by immunohistochemical staining for HGM, MUC6, MUC2 and CDI0, can be used to predict the pattern of gastric carcinoma recurrence after curative resection.

British Journal of Cancer (2004) 9 I, I342-1348. doi: I0.1038/sj.bjc.6602 I47 www.bjcancer.com

Published online 3I August 2004

(c) 2004 Cancer Research UK

Keywords: gastric carcinoma; immunohistochemistry; human gastric mucin; MUC6; MUC2; CDI 0; recurrence pattern

Gastric carcinoma is histologically classified in two manners, differentiated $v s$ undifferentiated, or intestinal $v s$ diffuse, based on the tendency of gland formation (Lauren, 1965; Nakamura et al, 1968). However, recent mucin histochemical and immunohistochemical examinations have demonstrated that gastric and intestinal phenotypic cell markers are widely expressed in gastric carcinomas, irrespective of their histologic type (Tatematsu et al, 1990; Egashira et al, 1999; Endoh et al, 1999, 2000; Koseki et al, 2000; Saito et al, 2001; Tajima et al, 2001a, b, 2003; Tsukashita et al, 2001; Kabashima et al, 2002; Shibata et al, 2003). Several authors have reported that gastric carcinomas can be classified as having either a gastric (G), gastric and intestinal mixed (GI) or intestinal (I) phenotype, depending on the immunopositivity of human gastric mucin (HGM), MUC6, MUC2 and CD10 stainings (Tajima et al, 2001b, 2003; Kabashima et al, 2002). Human gastric mucin, MUC6, MUC2 and CD10 specifically express in gastric

*Correspondence: Dr Y Tajima; E-mail: surgery@med.showa-u.ac.jp Received 2 April 2004; revised 5 July 2004; accepted 16 July 2004; published online 31 August 2004 surface mucous cells, pyloric gland cells, intestinal goblet cells of the mature gastrointestinal tract and brush border of intestinal epithelial cells, respectively. We previously reported that G-phenotype tumours accounted for $27.7 \%$ of differentiated tumours, often referred to as intestinal-type tumours according to Lauren (1965), while I-phenotype tumours accounted for $10.1 \%$ of undifferentiated tumours (Tajima et al, 2001b). The phenotypic marker expression of tumours is conventionally thought to imitate that of the tissue of origin. Thus, the previous data described above suggest that gastric carcinomas can occur in various types of gastric mucosa, although differentiated tumours have generally been considered to arise from gastric mucosa with intestinal metaplasia and undifferentiated tumours to arise from ordinary gastric mucosa without intestinal metaplasia (Lauren, 1965; Nakamura et al, 1968). Recently, G-phenotype tumours have been associated with poorer outcome and greater malignant potential in the incipient phase of invasion and metastasis, compared with those of other phenotype tumours (Endoh et al, 1999; Koseki et al, 2000; Saito et al, 2001; Tajima et al, 2001b; Kabashima et al, 2002; Shibata et al, 2003). Therefore, phenotypic classification could be 
useful not only for investigating the tumorigenesis of gastric carcinoma, but also for evaluating tumour aggressiveness. Phenotypic expression in gastric carcinomas has also been reported to be strongly dependent on genetic changes (Endoh et al, 2000).

Early tumour detection, standardised surgical treatment with systematic lymph node dissection and appropriate chemotherapy have improved the survival of patients with gastric carcinoma (Otsuji et al, 1998). However, recurrences are likely to assume a variety of forms in various different organs, even after a curative resection of the gastric carcinoma. Risk factors for peritoneal recurrence have been reported to include an undifferentiated histological type, infiltrative tumour growth and serosal invasion, while risk factors for haematogenous recurrence have been reported to include a vessel invasion, differentiated histological type and expansive tumour growth (Maehara et al, 1996; Yoo et al, 2000; Ohno et al, 2003; Roviello et al, 2003). The prediction of recurrence patterns after surgery could help better follow-up programmes and appropriate treatment strategies to be designed for gastric carcinoma patients. However, the relation between gastric and intestinal phenotypic marker expression in gastric carcinomas and the recurrence pattern after surgery has not been investigated.

In the present study, we examined surgically resected tumour specimens from 213 patients with advanced gastric carcinoma using HGM, MUC6, MUC2 and CD10 immunohistochemical stainings to clarify the relation between gastric and intestinal phenotypic marker expression in gastric carcinomas and the recurrence pattern after surgery.

\section{MATERIALS AND METHODS}

\section{Patients}

Our series consisted of 213 patients who had undergone potentially curative resections for advanced gastric carcinoma between 1994 and 1998 at the Showa University Hospital and the Toyosu Hospital, Showa University, Tokyo, Japan. A total gastrectomy, or a distal or proximal subtotal gastrectomy with lymph node dissection, was performed, depending on the tumour location and surgical margin. Each patient underwent D2 (141 cases) or D3 (72 cases) lymphadenectomy and a mean of $28.3 \pm 11.8$ lymph nodes per patient was dissected. As for the chemo or radiation therapies after surgery, all patients received oral administration of 5-fluorouracil (5-FU; $200 \mathrm{mg} \mathrm{body}^{-1} \mathrm{day}^{-1}$ ) for more than 2 months after the operation. None of the patients received radiotherapy after surgery. After operation, routine follow-up consisted of a physical examination, blood examination (including carcinoembryonic antigen, CA19-9 levels), radiological examination, endoscopic examination, computed tomography and ultrasonography. Follow-up examinations were performed every 3 months for the first postoperative year and then at intervals of between 6 and 12 months for at least 5 years. Of the 213 patients in our series, 116 patients were still alive with no recurrences at the time of the last follow-up (control group) and 97 had died from recurrent gastric carcinoma. The recurrence sites in the 97 patients were precisely determined by radiological examination, endoscopic examination, computed tomography, ultrasonography or scintigraphy, and the recurrence pattern was divided into three groups: peritoneal, haematogenous and locoregional recurrences. Peritoneal recurrence occurred in 60 patients, haematogenous recurrence occurred in 32 patients and locoregional recurrence occurred in 18 patients (Figure 1). Haematogenous recurrence occurred in the liver in 29 patients, in the lung in five patients and in the brain in three patients. Locoregional recurrence occurred in the lymph node, in 16 patients and in the anastomosis or stump in two patients.

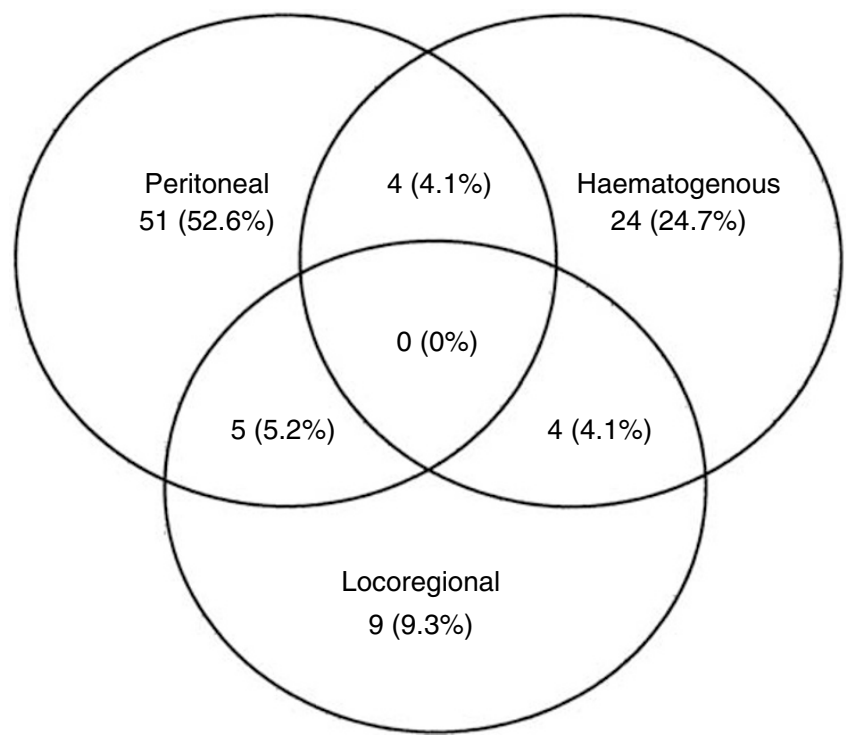

Figure I Recurrence patterns in 97 patients after curative resection.

\section{Clinicopathologic review}

Serial 5-mm-thick tissue sections of the entire tumour were prepared from the resected specimens fixed with $10 \%$ buffered formalin, embedded in paraffin and stained with haematoxylin and eosin (H\&E). Clinicopathologic findings such as age, gender, tumour size, tumour location, depth of invasion, growth pattern, lymphatic invasion, blood vessel invasion and lymph node metastasis were reviewed according to the Japanese Classification of Gastric Carcinoma (Japanese Gastric Cancer Association, 1998). In the present study, the tumours were classified histologically as either differentiated or undifferentiated, according to the criteria described by Nakamura et al (1968).

\section{Immunohistochemical staining}

The following mouse monoclonal antibodies were used: 45M1 (Novocastra Laboratories Ltd, UK), diluted 1:50, to detect HGM; CLH5 (Novocastra Laboratories Ltd), diluted 1:50, to detect MUC6 glycoprotein; Ccp58 (Novocastra Laboratories Ltd), diluted 1:100, to detect MUC2 glycoprotein; and 56C6 (Novocastra Laboratories Ltd), diluted $1: 40$, to detect CD10 glycoprotein expression. 45M1 and CLH5 were examined as G-phenotype markers, and Ccp58 and 56C6 were examined as I-phenotype markers. $45 \mathrm{M} 1$ recognises the mucin epitope located in the peptide core of HGM, which is synonymous with MUC5AC. This antibody is known to react with surface foveolar cells in the stomach (Bara et al, 1998; Nollet et al, 2002). MUC6 glycoprotein is expressed in mucous cells of the neck zone of oxyntic mucosa and in antral glands (De Bolos et al, 1995; Reis et al, 1999; Machado et al, 2000). MUC2 glycoprotein, also known as the 'intestinal-mucin-related protein antigen', is an intestinal apomucin and is known to be also expressed in the supranuclear area of the goblet cells in regions showing intestinal metaplasia in the stomach (Kim and Gum, 1995; Sakamoto et al, 1997; Baldus et al, 1998; Utsunomiya et al, 1998; Reis et al, 1999; Machado et al, 2000). CD10 glycoprotein is a $100-\mathrm{kDa}$ cell metalloendopeptidase that inactivates a variety of biologically active peptides and is known to be expressed on the brush border of intestinal epithelial cells as well as in the germinal centres of lymphoid follicles and the microvilli of the kidney (Ronco et al, 1984; Trejdosiewicz et al, 1985).The avidin-biotinyl-peroxidase complex immunohistochemical method was used for all immuno- 
A

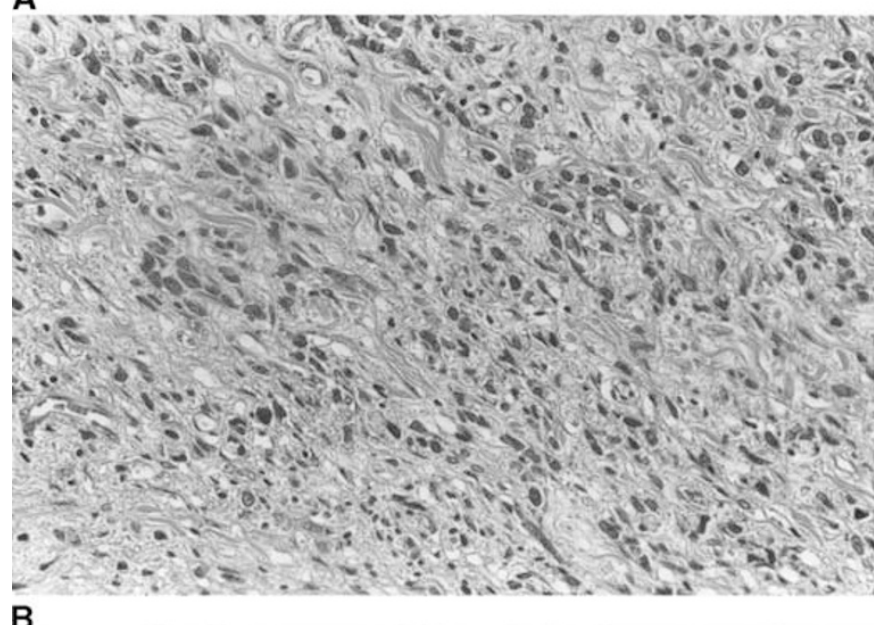

B

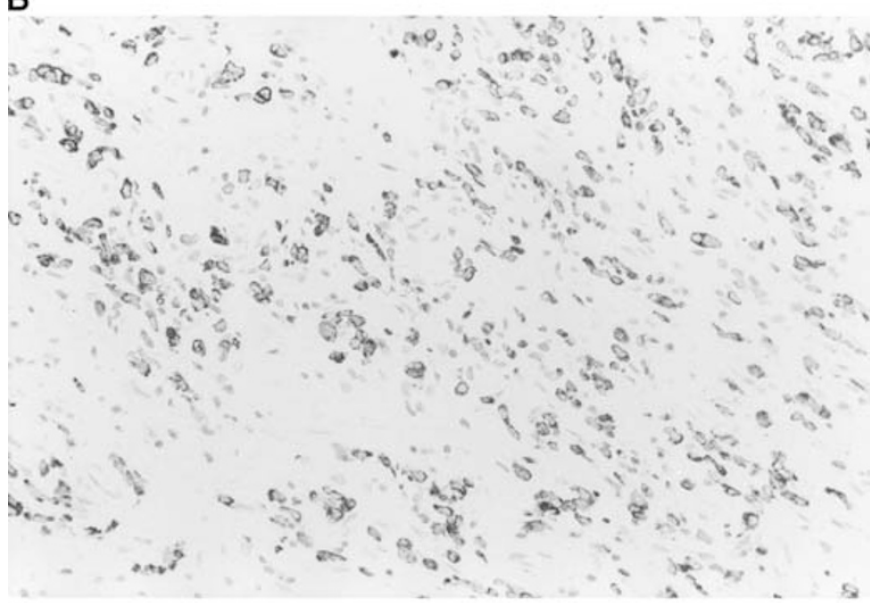

C

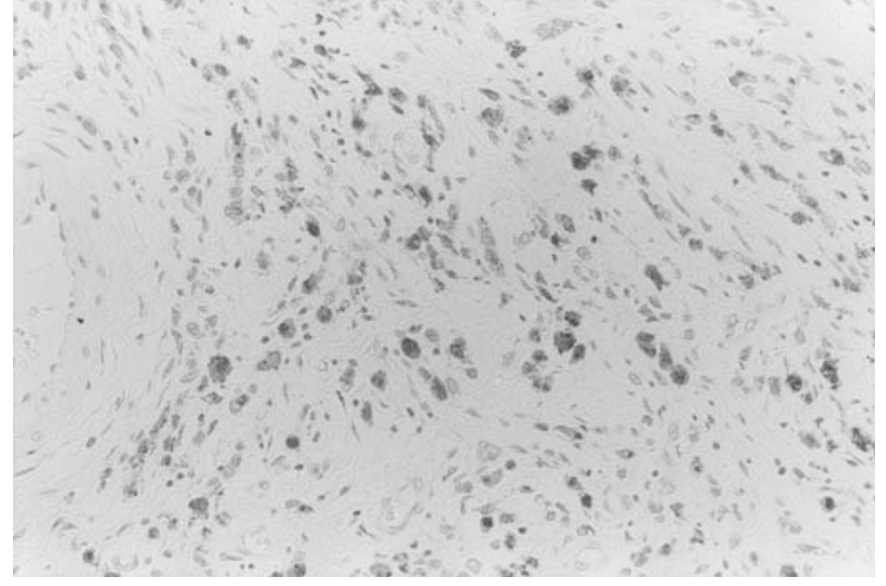

Figure 2 (A-C) Gastric carcinoma of the G-phenotype. (A) Histological features (H\&E, original magnification $\times 400)$. (B) Human gastric mucin is expressed in the cancer cell cytoplasm (45Ml, original magnification $\times 400$ ). (C) MUC6 glycoprotein is also expressed in the cancer cell cytoplasm $(\mathrm{CLH5}$, original magnification $\times 400)$.

histochemical studies according to a previously described protocol (Hsu et al, 1981).

With regard to the evaluations of HGM, MUC6, MUC2 and CD10 staining, distinct staining in more than $5 \%$ of the tumour cells was recorded as positive immunoreactivity for the relevant marker. These immunohistochemical methods were used to classify the tumours into four different phenotypes: tumours with Gphenotypic cells accounting for more than $5 \%$ of their cell
A

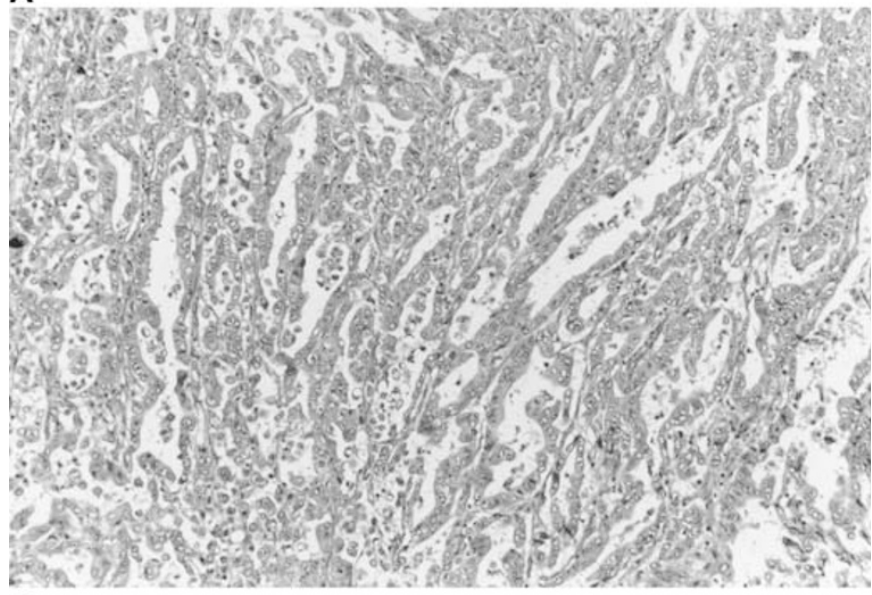

B
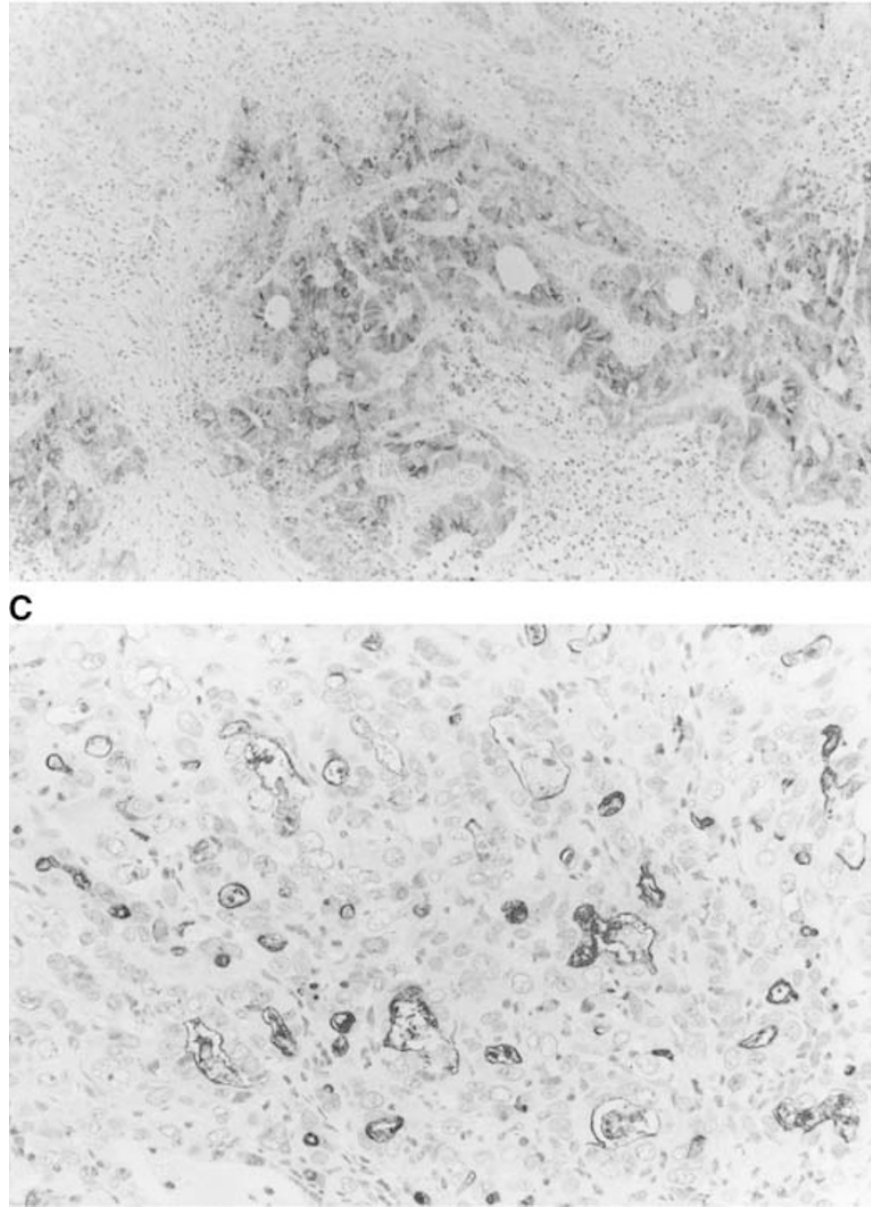

Figure 3 (A-C) Gastric carcinoma of the I-phenotype. Histological features (H\&E, original magnification $\times 200$ ). (B) MUC2 glycoprotein is expressed in the cancer cell cytoplasm (Ccp58, original magnification $\times 200$ ). (C) CD 0 glycoprotein is also expressed on the luminal surfaces of cancerous glands (56C6, original magnification $\times 200$ )

population were classified as G- phenotype carcinomas (Figure 2); those with I-phenotypic cells accounting for more than $5 \%$ of their cell population were classified as I-phenotype carcinomas (Figure 3); those with both gastric and intestinal phenotypic cells accounting for more than $5 \%$ of their cell population were classified as GI-phenotype carcinomas; and those with both gastric and intestinal phenotypic cells accounting for less than $5 \%$ of their cell population were regarded as carcinomas of 
UC-phenotype (Tajima et al, 2001b, 2003; Tsukashita et al, 2001; Yao et al, 2002).

The histopathological and immunohistochemical examinations were independently performed by two observers (Tajima and Yamazaki). The results were then compared, and any discrepancies were resolved by consensus after further histopathological review.

\section{Statistical analysis}

Statistical calculations were performed using the Stat View software package (version 5.0; Abacus Concepts, Inc., Berkeley, CA, USA). The data were analysed using Student' $t$-test or MannWhitney $U$-test, in addition to the $\chi^{2}$ test or Fisher' exact test. Differences of $P<0.05$ were considered significant.

\section{RESULTS}

\section{Relations between recurrence patterns and clinicopathological features}

The relations between the recurrence patterns and the clinicopathological features are shown in Table 1. Peritoneal recurrence was significantly associated with a larger tumour size $(P<0.0001)$, a deeper depth of invasion $(P<0.0001)$ and higher incidences of tumours with whole tumour location $(P=0.012)$, undifferentiatedtype tumours $(P=0.0036)$, infiltrative growth tumours $(P=0.0009)$ and lymph node metastasis $(P=0.0002)$, compared with the control group. Haematogenous recurrence was significantly associated with a higher mean age $(P=0.016)$, a higher male-to-female ratio $(P=0.02)$ and higher incidences of blood vessel invasion $(P=0.032)$ and lymph node metastasis $(P=0.0061)$, compared with the control group. No significant differences in the evaluated clinicopathological factors were observed between patients with locoregional recurrence and the control group.

\section{Relations between recurrence patterns and expressions of HGM, MUC6, MUC2 and CD10}

The relations between the recurrence patterns and the expressions of HGM, MUC6, MUC2 and CD10 are shown in Table 2. The expressions of HGM, MUC6, MUC2 and CD10 were observed in $73.3,61.7,30.0$ and $15.0 \%$ of patients in the peritoneum recurrence group, respectively; in $43.8,43.8,37.5$ and $43.8 \%$ of patients in the haematogenous recurrence group, respectively; in 66.7, 66.7, 50.0 and $16.7 \%$ of patients in the locoregional recurrence group, respectively; and in $54.3,62.9,61.2$ and $23.3 \%$ of patients in the control group, respectively. Peritoneal recurrence was significantly associated with HGM-positive tumours $(P=0.022)$ and MUC2negative tumours $(P=0.0002)$, compared with the control group. Haematogenous recurrence was significantly associated with MUC2-negative tumours $(P=0.028)$ and CD10-positive tumours $(P=0.039)$, compared with the control group. No significant differences in expression were observed between the locoregional recurrence group and the control group.

\section{Relations between recurrence patterns and the phenotypic marker expression patterns of the tumours}

The relations between the recurrence patterns and the phenotypic marker expression patterns of the tumours are shown in Table 3. G-, GI-, I- and UC-phenotype tumours composed 58.3, 26.7, 8.3 and $6.7 \%$ of the peritoneum recurrence group, respectively; 25.0 , $37.5,25.0$ and $12.5 \%$ of the haematogenous recurrence group, respectively; 44.4, 44.4, 11.1 and $0 \%$ of the locoregional recurrence group, respectively; and 28.4, 51.7, 15.5 and $4.3 \%$ of the control group, respectively. Peritoneal recurrence was significantly associated with G-phenotype tumours, compared with the control
Table I Relations between recurrence patterns and clinicopathological characteristics

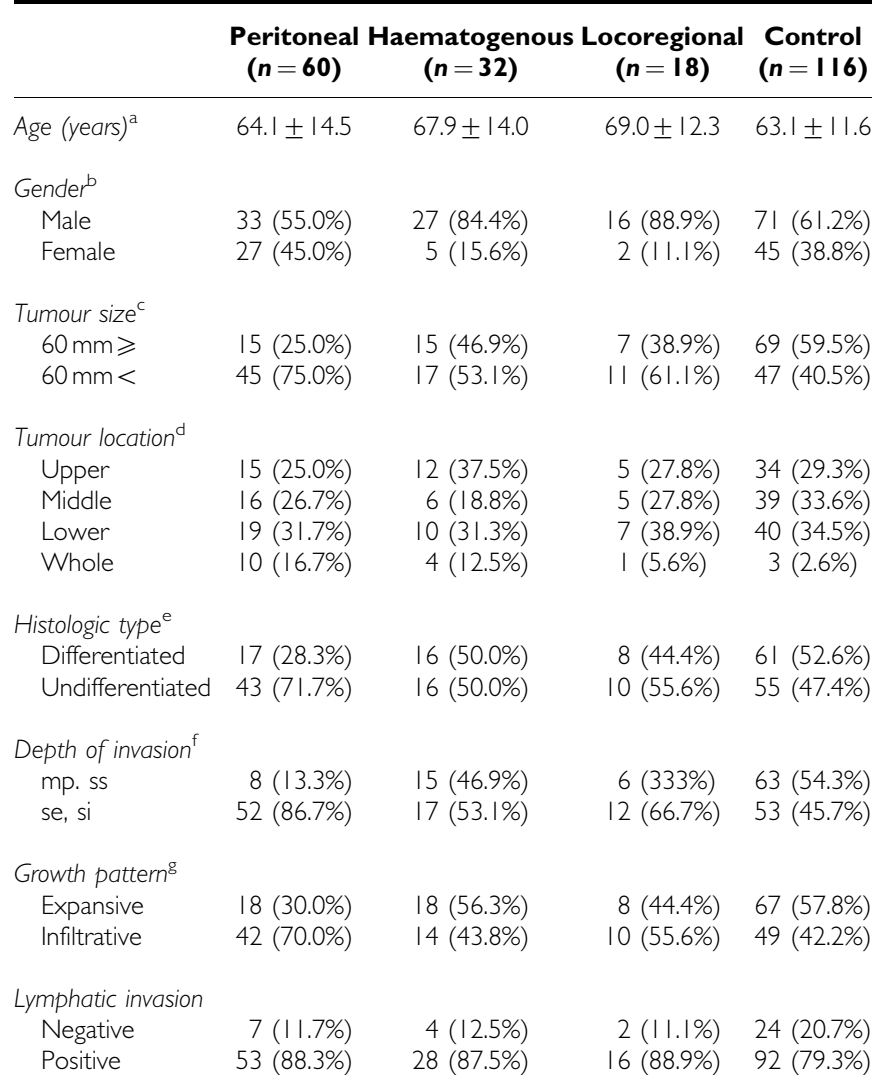

Blood vessel

invasion $^{\text {h }}$

$\begin{array}{lllll}\text { Negative } & 37(61.7 \%) & \text { II (34.4\%) } & 6(33.3 \%) & 67(57.8 \%)\end{array}$

$\begin{array}{lllll}\text { Positive } & 23(38.3 \%) & 21(65.6 \%) & 12(66.7 \%) & 49(42.2 \%)\end{array}$

Lymph node

metastasis ${ }^{i}$

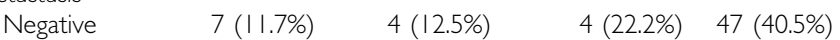

Positive $\quad 53(883 \%) \quad 28(87.5 \%) \quad 14(77.8 \%) \quad 69(59.5 \%)$

$\mathrm{mp}=$ muscularic propria; $\mathrm{ss}=$ subserasa; se = serosa exposed; si = serosa-infiltrating adjacent tissue. ${ }^{a} P=0.016$ (haematogenous vs control). ${ }^{b} P=0.0057$ (peritoneal vs haematogenous) and $P=0.02$ (haematogenous vs control). ${ }^{c} P<0.001$ (peritoneal vs control). ${ }^{d} p=0.012$ (peritoneal vs control). ${ }^{e} P=0.0036$ (peritoneal vs control). ${ }^{f} P=0.001$ (peritoneal vs haematogenous) and $P<0.000$ I (peritoneal vs control). ${ }^{g} P=0.026$ (peritoneal vs haematogenous) and $P=0.0009$ (peritoneal vs control). ${ }^{h} P=0.023$ (peritoneal vs haematogenous) and $P=0.032$ (haematogenous vs control). ${ }^{i} P=0.0002$ (peritoneal vs control) and $P=0.0061$ (haematogenous vs control).

group $(P=0.0002)$. No significant differences in the phenotypic marker expression patterns of tumours in the haematogenous or locoregional recurrence groups were found, when compared with the control group.

\section{DISCUSSION}

Several studies on the relationship between recurrence patterns of gastric carcinoma after surgery and clinicopathological characteristics have been performed (Maehara et al, 1996; Yoo et al, 2000; Ohno et al, 2003; Roviello et al, 2003). Reported risk factors for peritoneal recurrence include an undifferentiated histologic type, infiltrative tumour growth and serosal invasion (Maehara et al, 
1996; Yoo et al, 2000; Roviello et al, 2003). Reported risk factors for haematogenous recurrence, on the other hand, include blood vessel invasion, a differentiated histologic type and expansive tumour growth (Maehara et al, 1996; Yoo et al, 2000; Ohno et al, 2003). In the present study, peritoneal recurrence was significantly associated with a larger tumour size, a deeper depth of invasion, and higher incidences of tumours with whole tumour location, undifferentiated-type tumours, infiltrative growth tumours and lymph node metastasis, compared with the control group, while haematogenous recurrence was significantly associated with a higher mean age, a higher male-to-female ratio, and higher incidences of blood vessel invasion and lymph node metastasis. Therefore, clinicopathological analysis appears to be a useful approach predicting recurrence patterns after surgery. Furthermore, our present results clearly show that recurrence patterns after curative resection vary with the gastric and intestinal phenotypic marker expression of the tumour. To the best of our knowledge, this is the first report describing an association between phenotypic marker expression in gastric carcinomas and the recurrence pattern after surgery.

Our present results revealed that the incidences of HGMpositive tumours and MUC2-negative tumours were significantly higher in the peritoneal recurrence group than in the control group. Taking into account the expression combinations of HGM, MUC6, MUC2 and CD10, the incidence of G-phenotype tumours was significantly higher in the peritoneal recurrence group than in the control group. With respect to the relations between the

Table 2 Relations between recurrence patterns and expressions of human gastric mucin, MUC6, MUC2 and CDIO

\begin{tabular}{|c|c|c|c|c|}
\hline & $\begin{array}{c}\text { Peritoneal } \\
(n=60)\end{array}$ & $\begin{array}{l}\text { Haematogenous } \\
(n=32)\end{array}$ & $\begin{array}{l}\text { Locoregional } \\
\quad(n=18)\end{array}$ & $\begin{array}{l}\text { Control } \\
(n=116)\end{array}$ \\
\hline \multicolumn{5}{|c|}{$\begin{array}{l}\text { Human gastric } \\
\text { mucin }^{\mathrm{a}}\end{array}$} \\
\hline Negative & 16 (26.7\%) & 18 (56.3\%) & $6(33.3 \%)$ & 53 (45.7\%) \\
\hline Positive & 44 (73.3\%) & $14(43.8 \%)$ & $12(66.7 \%)$ & $63(54.3 \%)$ \\
\hline \multicolumn{5}{|l|}{ MUC6 } \\
\hline Negative & 23 (38.3\%) & I 8 (56.3\%) & $6(33.3 \%)$ & 43 (37.1\%) \\
\hline Positive & 37 (61.7\%) & $14(43.8 \%)$ & $12(66.7 \%)$ & 73 (62.9\%) \\
\hline \multicolumn{5}{|l|}{$M U C 2^{b}$} \\
\hline Negative & 42 (70.0\%) & 20 (62.5\%) & $9(50.0 \%)$ & 45 (38.8\%) \\
\hline Positive & 18 (30.0\%) & $12(37.5 \%)$ & $9(50.0 \%)$ & $7 \mid(61.2 \%)$ \\
\hline \multicolumn{5}{|l|}{$C D / 0^{c}$} \\
\hline Negative & $5 \mid(85.0 \%)$ & 18 (56.3\%) & 15 (83.3\%) & 89 (76.7\%) \\
\hline Positive & $9(15.0 \%)$ & $14(43.8 \%)$ & $3(16.7 \%)$ & 27 (23.3\%) \\
\hline
\end{tabular}

clinicopathological findings and HGM expression, we previously reported that HGM expression was correlated with a higher proportion of infiltrative tumours and undifferentiated-type tumours in advanced gastric carcinomas; we also found a higher incidence rate of infiltrative tumours in G-phenotype tumours than in I-phenotype tumours (Tajima et al, 2001b). Even differentiated gastric carcinomas with a G-phenotype have been reported to be more likely to transform into undifferentiated carcinomas and exhibit infiltrative growth to deeper layers of the invaded surrounding structures through the loss of E-cadherin function, compared with differentiated gastric carcinomas with an I-phenotype (Endoh et al, 1999; Saito et al, 2001; Tajima et al, 2001b). Undifferentiated carcinomas with infiltrative growth have been reported to be mainly noted in cases with an advanced level of invasion into the serosal layer or adjacent organs (Maehara et al, 1996). As described above, our present results showed that peritoneal recurrence was dominant among undifferentiated tumours and tumours exhibiting serosal invasion, infiltrative tumour growth and lymph node metastasis; these clinicopathological characteristics have also been correlated with G-phenotype and HGM-positive tumours (Tajima et al, 2001b). In these cases, the cancerous cells are thought to infiltrate the gastric wall, penetrate the serosa and disseminate trans-serosally to the peritoneum. Therefore, the association between G-phenotype or HGM-positive tumours and peritoneal recurrence in the present study may be attributed to the clinicopathological characteristics of the G-phenotype or HGM-positive tumours.

Haematogenous metastasis is thought to mainly occur when cancerous cells released from the primary site enter the blood vessel system and are transported to a target organ, where attachment and proliferation occurs (Maehara et al, 1996). Thus, blood vessel invasion is considered to be one of the most important factors for haematogenous metastasis. In the present study, the incidences of MUC2-negative tumours and CD10positive tumours were significantly higher in the haematogenous recurrence group than in the control group. Concerning the relations between the clinicopathological findings and MUC2 expression, Utsunomiya et al (1998) reported that MUC2 expression in tumours was correlated with lower levels of invasion and lymph node metastasis in gastric carcinomas. In the present study, peritoneal recurrence was also strongly associated with MUC2-negative tumours. Among colorectal carcinomas, MUC2positive tumours have been reported to have a relatively good prognosis, with a low incidence of liver metastasis (Hanski et al, 1997). Therefore, MUC2 expression might be inversely correlated with tumour aggressiveness in gastric carcinomas. As for the relations between the clinicopathological findings and CD10 expression, we previously reported that CD10 expression was correlated with a higher male-to-female ratio of patients, a differentiated histologic type, expansive tumour growth and blood vessel invasion in advanced gastric carcinomas (Tajima et al, 2001b); these clinicopathological characteristics have also been associated with haematogenous metastasis (Maehara et al, 1996; Yoo et al, 2000; Roviello et al, 2003). We also reported a higher

Table 3 Relations between recurrence patterns and the phenotypic marker expression pattern of the tumour

\begin{tabular}{|c|c|c|c|c|}
\hline & $\begin{array}{l}\text { Peritoneal } \\
(n=60)\end{array}$ & $\begin{array}{l}\text { Haematogenous } \\
\quad(n=32)\end{array}$ & $\begin{array}{l}\text { Locoregional } \\
\quad(n=18)\end{array}$ & $\begin{array}{l}\text { Control } \\
(n=116)\end{array}$ \\
\hline Gastric phenotype ${ }^{a}$ & $35(58.3 \%)$ & $8(25.0 \%)$ & $8(44.4 \%)$ & $33(28.4 \%)$ \\
\hline Intestinal phenotype & $5(8.3 \%)$ & $8(25.0 \%)$ & $2(11.1 \%)$ & $18(15.5 \%)$ \\
\hline
\end{tabular}

${ }^{a} P=0.0046$ (peritoneal vs haematogenous) and $P=0.0002$ (peritoneal vs control). ${ }^{b} P=0.0025$ (peritoneal vs control). 
incidence rate of synchronous liver metastasis in CD10-positive tumours than in CD10-negative tumours (Tajima et al, 2001b). Among colorectal carcinomas, CD10-positive tumours exhibited a higher incidence of blood vessel invasion and were at increased risk of liver metastais (Yao et al, 2002). Moreover, an ultrastructural study by Shimizu et al (2000) revealed that microvilli, as demonstrated by CD10 expression, were generated on the luminal surface of metastatic liver adenocarcinomas. Based on these previous data and the present findings, CD10-positive gastric carcinomas appear to have a strong tendency toward blood vessel invasion, leading to haematogenous metastasis.

In the present study, we demonstrated that the recurrence patterns after curative resection, such as peritoneal and haematogenous recurrences, vary with the phenotypic marker expression of the tumour. Therefore, evaluating the gastric and intestinal phenotypic marker expression of tumours may be useful for predicting the recurrence patterns of the gastric carcinomas after surgery. Careful postoperative follow-up is vital for patients with a high-risk peritoneal or haematogenous recurrence, since the prognosis of patients with recurrence is very poor; additional and intensive therapies after surgery may be indicated for such cases. The results of several randomised trials have demonstrated that intraperitoneal chemotherapy in normothermic or hyperthermic patients tends to improve survival rates and decrease the incidence of peritoneal failure compared with surgery alone (Yu et al, 1998; Fujimoto et al, 1999). For patients with a high risk of peritoneal recurrence, intraperitoneal chemotherapy may thus

\section{REFERENCES}

Baldus SE, Zirbes TK, Engel S, Hanisch FG, Monig SP, Lorenzen J, Glossmann J, Fromm S, Thiele J, Pichlmaier H, Dienes HP (1998) Correlations of the immunohistochemical reactivity of mucin peptide cores MUC1 and MUC2 with the histopathological subtype and prognosis. Int J Cancer 79: 133-138

Bara J, Chastre E, Mahiou J, Singh RL, Forgue-Lafitte ME, Hollande E, Godeau F (1998) Gastric M1 mucin, an early oncofetal marker of colon carcinogenesis, is encoded by the MUC5AC gene. Int J Cancer 75: $767-773$

De Bolos C, Garrido M, Real FX (1995) MUC6 apomucin shows a distinct normal tissue distribution that correlates with Lewis antigen expression in the human stomach. Gastroenterology 109: $723-734$

Egashira Y, Shimoda T, Ikegami M (1999) Mucin histochemical analysis of minute gastric differentiated adenocarcinoma. Pathol Int 49: 55-61

Endoh Y, Sakata K, Tamura G, Ohmura K, Ajioka Y, Watanabe H, Motoyama T (2000) Cellar phenotypes of differentiated-type adenocarcinomas and precancerous lesions of the stomach are dependent on the genetic pathways. J Pathol 191: 257-263

Endoh Y, Tamura G, Watanabe H, Ajioka Y, Motoyama T (1999) The common 18-base pair deletion at codons $418-423$ of the E-cadherin gene in differentiated-type adenocarcinomas and intramucosal precancerous lesions of the stomach with the features of gastric foveolar epithelium. J Pathol 189: $201-206$

Fujimoto S, Takahashi M, Mutou T, Kobayashi K, Toyosawa T (1999) Successful intraperitoneal hyperthermic chemoperfusion for the prevention of postoperative peritoneal recurrence in patients with advanced gastric carcinoma. Cancer 85: 529-534

Hanski C, Riede E, Gratchev A, Foss HD, Bohm C, Klussmann E (1997) MUC2 gene suppression in human colorectal carcinomas and their metastasis: in vitro evidence of the modulatory role of DNA methylation. Lab Invest 77: 685-695

Hsu SM, Raine L, Fanger H (1981) Use of avidin-biotin peroxidase complex $(\mathrm{ABC})$ in immunoperoxidase techniques: a comparison between $\mathrm{ABC}$ and unlabeled antibody (PAP) procedures. J Histochem Cytochem 29: $577-580$

Japanese Gastric Cancer Association (1998) Japanese classification of gastric carcinoma - 2nd English edition. Gastric Cancer 1: 10-24

Kabashima A, Yao T, Sugimachi K, Tsuneyoshi M (2002) Relationship between biologic behavior and phenotypic expression in intramucosal gastric carcinomas. Hum Pathol 33: 80-86 be a valuable adjuvant at operation or early in the postoperative period.

Obvious differences in the biological behaviour of tumours with different phenotypic marker expressions have been reported. Kabashima et al (2002) reported that G-phenotype tumours could potentially degrade the extracellular matrix through the overexpression of matrix metalloproteinases, compared with Iphenotype tumours. Shibata et al (2003) reported that the apoptotic index/proliferative index ratio was significantly lower in G-phenotype tumours than in I-phenotype tumours. We previously reported that patients with G-phenotype tumours have a poorer prognosis than those with I-phenotype tumours among patients with advanced gastric carcinoma (Tajima et al, $2001 \mathrm{~b})$. We also previously reported that postoperative chemotherapy with 5-FU was effective for patients with G-phenotype tumours, since the incidence of intratumoral expression of thymidylate synthase, the target enzyme of 5-FU, was significantly low in G-phenotype tumours (Tajima et al, 2003). These previous data and our present findings suggest that appropriate postoperative follow-up programmes and therapeutic approaches may differ according to the phenotypic marker expression of the tumour.

In conclusion, our present findings show that the gastric and intestinal phenotypic marker expression of the tumour, determined by the HGM, MUC6, MUC2 and CD10 expression patterns, may be used to predict the recurrence pattern of gastric carcinomas after curative resections.
Kim YS, Gum JR (1995) Diversity of mucin genes, structure, function, and expression. Gastroenterology 109: 999-1013

Koseki K, Takizawa T, Koike M, Ito M, Nihei Z, Sugihara K (2000) Distinction of differentiated type early gastric carcinoma with gastric type mucin expression. Cancer 89: 724-732

Lauren P (1965) The two histological main types of gastric carcinoma: diffuse and so-called intestinal-type carcinoma. Acta Pathol Microbiol Scand A 64: 31 - 49

Machado JC, Nogueira AM, Carneiro F, Reis CA, Sobrinho-Simoes M (2000) Gastric carcinoma exhibits distinct types of cell differentiation: an immunohistochemical study of trefoil peptides (TFF1 and TFF2) and mucins (MUC1, MUC2, MUC5AC, and MUC6). J Pathol 190: 437-443

Maehara Y, Emi Y, Baba H, Adachi Y, Akazawa K, Ichiyoshi Y, Sugimachi K (1996) Recurrences and related characteristics of gastric cancer. Br J Surg Cancer 74: 975 - 979

Nakamura K, Sugano H, Takagi K (1968) Carcinoma of the stomach in incipient phase: its histogenesis and histological appearances. Gann 59: $251-258$

Nollet S, Forgue-Lafitte ME, Kirkham P, Bara J (2002) Mapping of two new epitopes on the apomucin encoded by MUC5AC gene: expression in normal GI tract and colon tumors. Int J Cancer 99: 336-343

Ohno S, Fujii T, Ueda S, Nakamoto T, Kinugasa S, Yoshimura H, Tachibana M, Kubota H, Kumar Dhar D, Nagasue N (2003) Predictive factors and timing for liver recurrence after curative resection of gastric carcinoma. Am J Surg 185: 258-263

Otsuji E, Yamaguchi T, Sawai K, Hagiwara A, Taniguchi H, Takahashi T (1998) Recent advances in surgical treatment have improved the survival of patients with gastric carcinoma. Cancer 82: 1233-1237

Reis CA, David L, Correa P, Carneiro F, De Bolos C, Garcia E, Mandel U, Clausen H, Sobrinho-Simoes M (1999) Intestinal metaplasia of human stomach displays distinct pattern of mucin (MUC1, MUC2, MUC5AC, and MUC6) expression. Cancer Res 59: $1003-1007$

Ronco P, Allegri L, Melcion C, Pirotsky E, Appay MD, Bariety J, Pontillon F, Verroust $\mathrm{P}$ (1984) A monoclonal antibody to brush border and passive nephritis. Clin Exp Immunol 55: 319-332

Roviello F, Marrelli D, Manzoni G, Morgagni P, Di Leo A, Saragoni L, De Stefano A, Italian Research Group for Gastric Cancer (2003) Prospective study of peritoneal recurrence after curative surgery for gastric cancer. Br J Surg 90: 1113-1119 
Saito A, Shimoda T, Nakanishi Y, Ochiai A, Toda G (2001) Histologic heterogeneity and mucin phenotypic expression in early gastric cancer. Pathol Int 51: $165-171$

Sakamoto H, Yonezawa S, Utsunomiya T, Tanaka S, Kim YS, Sato E (1997) Mucin antigen expression in gastric carcinomas of young and old adults. Hum Pathol 28: 1056-1065

Shibata N, Watari J, Fujiya M, Tanno S, Saitoh Y, Kohgo Y (2003) Cell kinetics and genetic instabilities in differentiated type early gastric cancers with different mucin phenotype. Hum Pathol 34: 32-40

Shimizu S, Yamada N, Sawada T, Ikeda K, Nakatani K, Seki S, Kaneda K, Hirakawa K (2000) Ultrastructure of early phase of hepatic metastasis of human colonic carcinoma cells with special reference to desmosomal junctions with hepatocyte. Pathol Int 50: 953-959

Tajima Y, Nakanishi Y, Yoshino T, Kokawa A, Kusano M, Shimoda T (2001a) Clinicopathological study of early adenocarcinoma of the gastric cardia: comparison with early adenocarcinoma of the distal stomach and esophagus. Oncology 61: 1-9

Tajima Y, Shimoda T, Nakanishi Y, Yokoyama N, Tanaka T, Shimizu K, Saito T, Kawamura M, Kusano M, Kumagai K (2001b) Gastric and intestinal phenotypic marker expression in gastric carcinomas and their prognostic significance: immunohistochemical analysis of 136 lesions. Oncology 61: $212-220$

Tajima Y, Shimoda T, Nakanishi Y, Yokoyama N, Tanaka T, Shimizu K, Saito T, Kawamura M, Kusano M, Kumagai K (2003) Association of gastric and intestinal phenotypic marker expression of gastric carcinomas with tumor thymidylate synthase expression and response to postoperative chemotherapy with 5-fluorouracil. J Cancer Res Clin Oncol 129: $683-690$

Tatematsu M, Ichinose M, Miki K, Hasegawa R, Kato T, Ito N (1990) Gastric and intestinal phenotypic expression of human stomach cancers as revealed by pepsinogen immunohistochemistry and mucin histochemistry. Acta Pathol Jpn 40: 494-504

Trejdosiewicz LK, Malizia G, Oakes J, Losowsky MS, Janossy G (1985) Expression of common acute lymphoblastic leukaemia antigen (CALLA gp100) in the brush border of normal jejunum and jejunum of patients with coeliac disease. J Clin Pathol 38: $1002-1006$

Tsukashita S, Kushima R, Bamba M, Sugihara H, Hattoti T (2001) MUC gene expression and histogenesis of adenocarcinoma of the stomach. Int J Cancer 94: 166-170

Utsunomiya T, Yonezawa S, Sakamoto H, Kitamura H, Hokita S, Aiko T, Tanaka S, Irimura T, Kim YS, Sato E (1998) Expression of MUC1 and MUC2 mucins in gastric carcinoma: its relationship with the prognosis of the patients. Clin Cancer Res 4: $2605-2614$

Yao T, Takata M, Tutsumi S, Nishiyama K, Taguchi K, Nagai E, Tsuneyoshi M (2002) Phenotypic expression of gastrointestinal differentiation markers in colorectal adenocarcinomas with liver metastasis. Pathology 34: $556-560$

Yoo CH, Noh SH, Shin DW, Choi SH, Min JS (2000) Recurrence following curative resection for gastric carcinoma. Br J Surg 87: 236-242

Yu W, Whang I, Suh I, Averbach A, Chang D, Sugarbaker PH (1998) Prospective randomized trial of early postoperative intraperitoneal chemotherapy as an adjuvant to resectable gastric cancer. Ann Surg 228: $347-354$ 